\title{
ENTRY PATTERNS OVER THE PRODUCT LIFE CYCLE ${ }^{\dagger}$
}

\author{
Óscar Gutiérrez Arnaiz \\ University of Zaragoza (Spain) \\ Francisco Ruiz-Aliseda \\ Northwestern University (USA)
}

October 2003

\begin{abstract}
We study an oligopoly model of entry over the product life cycle based on empirical evidence of demand for a new product growing over time and eventually falling. Yet, we assume that firms do not know ex ante when this can occur, which creates incentives to update information by delaying irreversible entry. Our model distinguishes and explains different patterns of entry characterized by firms entering simultaneously and/or in a sequential fashion, with entry rates accelerating or decelerating under certain conditions related to the rate at which individual profit decreases as more firms enter the industry.
\end{abstract}

Key words: Product Life Cycle, Real Options, Bayesian Updating, Open-loop Equilibria, and Accelerating Entry Rates.

JEL Classification: C73, D43, D92, L13, M30.

\footnotetext{
${ }^{\dagger}$ We are grateful to Anne Coughlan, James Dana, Daniel Spulber and participants at the $29^{\text {th }}$ EARIE Conference and the 2003 North American Summer Meeting of the Econometric Society for helpful comments. We also thank Vicente Salas-Fumás for his outstanding advice, profound insights and encouragement throughout this project. Óscar Gutiérrez and Francisco Ruiz-Aliseda gratefully acknowledge financial support from Research Project PO75/99-E and Fundación la Caixa, respectively. The usual disclaimer applies.

E-mail: franruiz@kellogg.northwestern.edu
} 


\section{INTRODUCTION}

There is numerous empirical evidence that shows that demand for some products grows over time until the market reaches its maturity, and gradually decays thereafter. The so-called theory of the product life cycle (PLC) provides a foundation to this pattern of consumer behavior based on diffusion models (Bass 1969, or Dodson and Muller 1978). ${ }^{1}$ This pattern of intertemporal changes in demand has certainly become a stylized fact for certain products such as durable goods. ${ }^{2}$ Indeed, many leading companies such as IBM, Sears, HewlettPackard or Eastman Kodak have used the pattern of sales predicated by the PLC theory for forecasting purposes (Bass 1980).

The nature of demand evolution over time is a crucial element when deciding whether or not to enter a market, and its role has been extensively studied in settings characterized by uncertainty. ${ }^{3}$ However, the patterns of entry triggered by the specific evolution of demand predicated by the PLC literature have not been examined in sufficient detail, at least at a theoretical level. ${ }^{4}$ In practice, uncertainty about the shape of the life cycle is critical for

\footnotetext{
${ }^{1}$ In the industrial organization literature, the theory of the (supply side) PLC refers to a different phenomenon, in particular, to the patterns of entry and exit of an industry based on intertemporal changes on supply side factors such as innovation capabilities or diffusion of technological knowledge among firms (Gort and Klepper 1982). This literature (e.g., Jovanovic 1982, or Hopenhayn 1992) has developed comprehensive models of industry evolution that focus on competitive selection of firms over time, and systematically ignores demand side aspects, as well as oligopoly settings, both of which will be the main focus of this paper.

${ }^{2}$ For example, see Grant (1998, Chapter 10), Kotler (1999, Chapter 10), and Pisano and Wheelwright (1995) for a discussion of the existence of PLCs and its implications. For empirical evidence and testing of their existence and shape, see Bass (1969, 1980), Brockhoff (1967), Kwoka (1996), Polli and Cook (1969) and Tsurumi and Tsurumi (1980).

${ }^{3}$ See Dixit and Pindyck (1994) for an updated treatment of investment under uncertainty. In particular, Chapter 5 studies the monopoly case, while Chapter 9 examines the duopoly case.

${ }^{4}$ See Lilien and Yoon (1990) for a pioneering empirical analysis.
} 
decision making insofar it is difficult to predict the various stages through which a product will go, as well as their duration. As a result, the pattern of demand growth over time can be assumed to be unknown to firms at the time they have to choose their product launching (or more generally entry) strategies. ${ }^{5}$ For this reason, the primary purpose of this paper is to propose a theoretical framework to characterize and explain various dynamics of entry into a market whose demand follows an unknown life cycle. The basic ingredients of the analysis are uncertainty about the PLC, strategic interactions among a finite number of firms, and irreversibility of entry. The assumptions that investment decisions made by firms are irreversible and demand for their product follows an unknown life cycle allows us to model entry decisions as the modern theory of irreversible investment under uncertainty does. ${ }^{6}$ The fact that a firm can delay its entry time allows for the treatment of investment opportunities as real options that can be exercised at any instant of time. In addition, the interaction between the entry decisions of all firms requires the use of game-theoretic arguments.

Given this setup, the model we employ provides a theoretical rationale for different entry patterns over the PLC. Thus, when the initial size of the market is relatively small, entry may occur in a sequential fashion, although the exact number of firms that enter the market would be contingent on the duration of the PLC. For intermediate market sizes, a certain number of entrants would enter simultaneously at the outset of the game and then no more firms would enter if the product turned out to have a short cycle. A successful product would attract additional entrants sequentially as the market gradually expanded until it began its recession. Finally, very large initial market sizes would lead firms not to care about the (unknown) future evolution of the market, despite it may turn out to be too small to

\footnotetext{
${ }^{5}$ For example, Tsurumi and Tsurumi (1980) provide an econometric model in which the transition from one stage of the PLC to another is due to gradual shifts in key demand parameters (e.g., income or price elasticity) whose date of occurrence is unknown.
} 
accommodate all of them. As a result, all firms would enter simultaneously at the initial date. In addition, the analysis yields a necessary and sufficient condition for accelerating and decelerating entry rates that are frequently observed in the early stages of market evolution. ${ }^{7}$ In particular, rates accelerate or decelerate depending on whether the percentage reduction in profit due to the immediate follower's entry decreases or increases with the number of remaining entrants.

There are a few previous papers that consider investment in a PLC setting, although their object of study is very different from ours. In the first place, Londregan (1990) examines the patterns of entry and exit of two firms with different capacities in a deterministic setting, and focuses on preemption and war of attrition issues. In the second place, Bollen (1999) considers a stochastic life cycle and shows that real options models that are based on a geometric brownian motion overvalue (undervalue) the option to expand (contract) a project. In the third place, Lilien and Yoon (1990) perform an empirical analysis of the tension that exists between the risks of premature entry over the PLC and the problem of missed opportunities due to a delay in entry. Appendix B in their paper introduces a simple discrete-time dynamic programming model that focuses on the optimal entry time of a monopolist over the PLC, but they do not endogenize market structure. In the last place, our paper builds on the novel real options framework introduced by Gutiérrez Arnaiz and RuizAliseda (2003), which is simpler than that of Bollen (1999). Its main feature is that the control space (i.e., time of investment) coincides with the state space (i.e., time of market maturity), which greatly simplifies an analysis that otherwise would be very complicated in

\footnotetext{
${ }^{6}$ For an excellent survey on this topic, see Dixit and Pindyck (1994).

${ }^{7}$ For instance, Gort and Klepper (1982) show that net entry rates accelerate and decelerate during certain phases of industry evolution.
} 
the oligopoly case, the focus of this paper. ${ }^{8}$ In this sense, the present work is the gametheoretic extension of the monopoly analysis in Gutiérrez Arnaiz and Ruiz-Aliseda (2003).

The remainder of the paper is organized as follows. Section 2 presents the model as well as its assumptions. Section 3 solves it and characterizes the entry patterns over the PLC. Section 4 briefly extends the basic model. Section 5 makes concluding remarks. The proofs of the propositions are simple but somewhat tedious, so they are relegated to an appendix.

\section{FOUNDATIONS OF THE THEORETICAL MODEL}

Let time, denoted by $t$, be a continuous variable, with $t \in[0, \infty)$. Suppose that at date $t=0$ a group of firms has to decide when to introduce a new product for which there already exists some latent demand. The number of firms is finite and is denoted by $n \geq 2 .{ }^{9} \mathrm{We}$ also assume that firms cannot perform technological improvements upon the product or the production technology. ${ }^{10}$

All firms face uncertainty about the temporal evolution of demand for the product when making their entry decisions. ${ }^{11}$ Uncertainty is assumed to unravel partially over time and demand evolves in the following manner. In a first stage, market size, which is positive at

\footnotetext{
${ }^{8}$ For instance, Bollen (1999) has to resort to numerical methods in a monopoly context.

${ }^{9}$ The finite number of firms seems an appropriate assumption, as suggested by Klepper and Graddy (1990, p. 36), because of expertise requirements.

${ }^{10}$ Certainly, it would be reasonable to assume that imitators have to develop over time the necessary capabilities to serve the market. Indeed, according to some authors such as Gort and Klepper (1982, p. 651), it is precisely the diffusion rate of technological knowledge and the possibility to innovate that mainly explains the empirical patterns of entry into an industry. In reality, all of these aspects and the ones we are considering come together and a more complete analysis should definitely integrate them to examine how they interact.

${ }^{11}$ For example, firms may not know some parameters of the diffusion process and may have to estimate them over time, leading to uncertainty about the PLC they face.
} 
date $t=0$, grows exponentially. This would represent the introduction and growth phases in the traditional PLC framework. However, the market reaches its ephemeral maturity at date $\tau$, where $\tau$ is a random variable with commonly known density $f(\tau)$ defined on $[0, \infty)$. We will slightly abuse the notation and $\tau$ will also denote its realization. Hence, in a second stage whose beginning is uncertain at date $0,{ }^{12}$ the size decreases exponentially and converges to 0 as $t \rightarrow \infty$, because consumers perceive that new substitutes of the product can serve their needs better. This decaying behavior would represent the decline stage of the product, as well as its subsequent gradual disappearance.

Formally, we make the following assumptions:

Assumption 1: Given $\tau$, the size of the market $a(\cdot)$ evolves over time as follows:

$$
a(t, \tau)= \begin{cases}A \exp \left(\frac{\alpha t}{2}\right) & \text { if } 0 \leq t \leq \tau \\ A \exp \left(\frac{\alpha(2 \tau-t)}{2}\right) & \text { if } t>\tau\end{cases}
$$

$A>0$ denotes the initial market size, whereas $\frac{\alpha}{2}>0$ denotes the growth rate of the market size (if the market is in expansion; otherwise, it is its decay rate). ${ }^{13}$

Assumption 2: The instant of maturity of the market $\tau$ is a random variable with continuous density function $f(\tau)$ with support contained in $(0, \infty)$.

\footnotetext{
${ }^{12}$ Arguably, we also assume that there are no detection lags regarding the random maturity date.

${ }^{13}$ Our assumption that the market increases at the same rate as it decreases is not important for our qualitative results. Also note that for simplicity the growth rate is exogenous, whereas in a more general model it should depend on the incumbent firms' actions to some extent, perhaps on advertising costs or some other marketing variable. One could go even further and assume that firms can make some decisions (for instance, promotions) to increase the size of the market once it has begun to decay. This more general analysis is beyond the scope of this paper.
} 
Figure 1 graphically illustrates two possible realizations of this kind of PLC. Despite it intends to serve as a "proxy" for the standard PLC, Kotler (1999, Chapter 10) reports that cycles such as the one assumed exist for products that are considered to be "fads", whose demand grows as quickly as it decreases and there is no maturity. The exponential growth and decline assumption could be considerably relaxed to get most results, but it are very useful when identifying the factor that is critical for explaining why entry rates are accelerating or decelerating.

\section{[FIGURE 1]}

In turn, Assumption 3 deals with the relationship between $a(t, \tau)$ and instantaneous profits made by firms active in the market. In particular, we implicitly assume that firms compete à la Cournot at any instant of time and are risk-neutral, so they maximize expected payoffs. Also, the instantaneous demand function is linear and unit variable costs are zero: ${ }^{14}$ Assumption 3: If $i$ firms are active in the market at date $t$, then each makes instantaneous profits of $\pi(i, t, \tau)=\left(\frac{a(t, \tau)}{i+1}\right)^{2}$

It is also natural to assume that investments are completely irreversible, which makes risk-taking a potentially relevant factor in explaining the patterns of entry:

Assumption 4: Any entrant bears a sunk cost of entry $K>0{ }^{15}$ and discounts future payoffs at the risk-free interest rate $r \geq 0$.

\footnotetext{
${ }^{14}$ Assumption 3 is relaxed in section 4.

${ }^{15}$ One may think of costs of setting up a distribution channel or the creation of brand equity through advertising expenditure, for example.
} 
Henceforward, denote the expectation operator conditional upon information at date $t$ by $E_{t}(\cdot)$ (the expectation is taken over $\tau$ ). Assumption 5 below ensures that the expected discounted value of one dollar that is capitalized at an instantaneous rate of $\alpha$ is finite no matter what the duration of the ascending phase of the PLC is. This bounds the value of the firms' investment opportunities.

Assumption 5: $E_{t}\left(e^{(\alpha-r) \tau}\right)<\infty \forall t \geq 0$.

\section{RESOLUTION OF THE MODEL}

We employ the methodology of optimal stopping games in order to solve the dynamic game with incomplete information. Throughout this section, we use the solution concept of openloop pure-strategy Nash equilibrium, ${ }^{16}$ and thus, without any loss of generality, we let the firm's index denote the position of entry no matter whether the market is growing or not. Hence, we first analyze firm n's optimal entry strategy, assuming that it invests at a later time than firm $n-1$. After obtaining firm $n$ 's optimal strategy we will proceed analogously with the preceding $n-1$ firms, taking into account that these firms know the followers' entry time.

In an open-loop equilibrium, firms must commit at $t=0$ to their entry times (see Reinganum 1981), so they cannot change their entry strategy even if they have incentives to preempt competitors as the game unfolds. For this reason, equilibria of this sort are also labeled "precommitment equilibria" (following Fudenberg and Tirole 1985). Note that there will be $n$ ! identical equilibria of this kind, interchanging the indexes of all the firms. Using this solution concept implies that firms can avoid coordination failures when entering.

\footnotetext{
${ }^{16}$ Unfortunately, the closed-loop equilibrium may not exist if there are more than two entrants as shown by Fudenberg and Tirole (1985). It is easy to characterize the closed-loop equilibrium for two firms, which involves rent equalization as it is usual in these kind of games.
} 
Firm $i$ 's problem at time $t=0, \quad i=1, \ldots, n$, is to choose an entry rule $T_{i}(\cdot)$ that maximizes its expected discounted stream of cash flows conditional upon information available at the time of entry. More generally, we have $T_{i}:[0, \infty) \times\{0,1\} \rightarrow\{\operatorname{In}$, Out $\}$, that is, firm $i$ 's strategy assigns a decision of whether to be in the market ("In") or not ("Out") to every instant of time depending on whether $\tau$ has been revealed to the firm at that time or not (events that are denoted by " 1 " and "0", respectively).

First of all, we proceed to characterize some of the common properties of the equilibrium strategies of all firms for the possible states of the system. Given our assumptions (no scrap value and positive profit-margin at any possible situation and date), one of the properties of these functions is that firm $i$ stays in the market forever once it has entered. This holds no matter if $\tau$ is known or not. In turn, Lemma 1 below describes the firms' behavior once the maturity of the market has been reached. According to this result, firms prefer to invest immediately once $\tau$ is revealed, but only if such date is sufficiently large (depending on the firm's index); otherwise, they prefer not to invest:

Lemma 1: It is optimal for firm $i(i=1, \ldots, n)$ to invest immediately at the revealed maturity date $\tau \forall \tau \geq t_{i}^{\max }=\max \left[0, \frac{1}{\alpha} \log \left(\frac{K(\alpha+r)(i+1)^{2}}{A^{2}}\right)\right]$, while investment during the declining phase of the market is not profitable $\forall \tau<t_{i}^{\max }$.

To characterize the equilibrium outcome fully, it only remains to focus on the optimal time $t_{i}^{*}$ at which firm $i(i=1, \ldots, n)$ would enter were the cycle in its ascending phase (i.e., when $\tau$ is not known yet). We first derive firm $n$ 's entry rule when $\tau$ has not been realized yet and all preceding firms have chosen to enter at $t_{i}^{*}, i=1, \ldots, n-1$. Using Lemma 1 , the value of firm $n$ 's investment opportunity at date $t=0$ as a function of its entry time $t_{n} \geq 0$ is: 


$$
\begin{aligned}
V_{n}\left(t_{n}\right)= & \int_{0}^{t_{n}} f(\tau) \max \left(0, \int_{\tau}^{\infty} \pi(n, s, \tau) e^{-r s} d s-K e^{-r \tau}\right) d \tau+ \\
& \int_{t_{n}}^{\infty} f(\tau)\left(\int_{t_{n}}^{\infty} \pi(n, s, \tau) e^{-r s} d s-K e^{-r t n}\right) d \tau
\end{aligned}
$$

If firm $n$ chooses to wait until $t_{n}$, then, for realizations smaller than $t_{n}$, it seizes the payoff to immediate investment at $\tau$ if and only if it is non-negative. In contrast, if firm $n$ ends up entering at $t_{n}$ while the product life cycle is growing, then it seizes an expected payoff that is conditional upon the information at time $t_{n}$ (namely, that $\tau$ must be greater than $\left.t_{n}\right)$. Note that, by another application of Lemma $1, V_{n}\left(t_{n}\right)$ can be rewritten as follows:

$$
V_{n}\left(t_{n}\right)= \begin{cases}\int_{t_{n}}^{\infty} f(\tau)\left(\int_{t_{n}}^{\infty} \pi(n, s, \tau) e^{-r s} d s-K e^{-r t_{n}}\right) d \tau & \text { if } t_{n} \in\left[0, t_{n}^{\max }\right) \\ \int_{t_{n}^{\max }}^{t_{n}} f(\tau)\left(\int_{\tau}^{\infty} \pi(n, s, \tau) e^{-r s} d s-K e^{-r \tau}\right) d \tau+ & \\ \int_{t_{n}}^{\infty} f(\tau)\left(\int_{t_{n}}^{\infty} \pi(n, s, \tau) e^{-r s} d s-K e^{-r t_{n}}\right) d \tau & \text { else }\end{cases}
$$

$V_{n}\left(t_{n}\right)$ can be easily shown to be continuously differentiable, so solving for firm $n$ 's optimal entry time amounts to solving the following:

$$
\begin{aligned}
\max _{t_{n}} & V_{n}\left(t_{n}\right) \\
\text { s.t. } & \int_{t_{n}}^{\infty} f(\tau)\left(\int_{t_{n}}^{\infty} \pi(n, s, \tau) e^{-r s} d s-K e^{-r t_{n}}\right) d \tau \geq 0 \\
& t_{n} \geq t_{n-1}^{*}
\end{aligned}
$$

The non-negativity constraint requires firm $n$ to make a profitable investment, in expectation, when the market is still growing. We have omitted the ex post non-negativity constraint because it is directly satisfied. Further, Lemma 1 has additional implications for the ex ante constraint, since letting $t_{n}=t_{n}^{\max }$ would automatically satisfy the constraint. Put differently, the lemma implies that there exists an instant of time $t_{n}^{\max }$ such that, if the market kept on 
growing, profits would be so large that even firm $n$ would find it profitable to enter the industry even though the market began to decay right after entry. Hence, the value of firm $n$ 's option to invest when the PLC is growing can never be negative at the optimum, and thus the constraint can be dropped from the firm's optimization program. However, we will solve the following program:

$$
\begin{aligned}
& \max _{t_{n}} V_{n}\left(t_{n}\right) \\
& \text { s.t. } t_{n} \geq 0
\end{aligned}
$$

$\mathrm{P} 2$ is more relaxed than P1, so we will have to check that its solution solves P1. We will use this solution procedure for all firms and check later on that it is indeed correct (see Lemma $4) .^{17}$

Now we can examine firm $n$ 's optimal strategy, but let us first introduce some new notation. More specifically, let $\lambda(t)=\frac{f(t)}{\int_{t}^{\infty} f(\tau) d \tau}$ denote the hazard rate, that is, the instantaneous probability of the PLC reaching its maturity at date $t$ given that this event has not occurred previously. As shown empirically by Barbarino and Jovanovic (2003), it sometimes can be natural to assume that the demand hazard rate is a non-increasing function, so let us assume this holds. ${ }^{18}$ This ensures that $V_{n}(\cdot)$ is single-peaked, so we can characterize firm $n$ 's entry rule in a precommitment equilibrium:

\footnotetext{
${ }^{17}$ It is worth noting that throughout we abuse the notation and use the same symbols for the candidate equilibrium entry rules and the actual ones.

${ }^{18}$ Log-convexity of the density function implies non-increasing hazard rate. Familiar probability distributions with log-convex density and support $(0, \infty)$ include the exponential, gamma and Weibull (in the latter two cases if the shape parameter is no larger than 1) and the F-distribution with the first degree of freedom no larger than 2. This condition could be relaxed to allow for distributions with an increasing hazard rate for some ranges, such
} 
Lemma 2: Firm n's optimal strategy is "enter at $t=t_{n}^{e}$ if $t \leq \tau$; else, do not enter" if there exists a unique $t_{n}^{e} \in\left(0, t_{n}^{\max }\right)$ such that

$$
e^{\alpha t_{n}^{e}}\left(1+\frac{\alpha}{r+\lambda\left(t_{n}^{e}\right)}\right)=\frac{K(\alpha+r)(n+1)^{2}}{A^{2}} .
$$

Firm n's optimal strategy is "enter at $t=0$ if $t \leq \tau$; else, do not enter" if such $t_{n}^{e}$ does not exist.

\section{Proof: See Appendix}

Therefore, Lemma 2 implies that a unique $t_{n}^{*}$ exists, with $t_{n}^{*} \in\left\{0, t_{n}^{e}\right\}$. Also, no entry by firm $n$ will occur in equilibrium during the declining stage of the PLC, since $t_{n}^{e}<t_{n}^{\max }$. Finally, we can also make the following observation: ${ }^{19}$

Remark 1: If $\tau$ has not been realized yet and the optimal entry time $t_{n}^{e}$ exists, then it is such that

$$
\frac{A^{2} e^{\alpha t_{n}^{e}}}{(n+1)^{2}} d t=r K d t+\lambda\left(t_{n}^{e}\right)\left(K-\int_{t_{n}^{e}}^{\infty} \frac{A^{2} e^{\alpha\left(2 t_{n}^{e}-s\right)}}{(n+1)^{2}} e^{-r\left(s-t_{n}^{e}\right)} d s\right) d t
$$

If the life cycle is still growing and the solution to the program is interior, then firm $n$ decides to enter at the instant of time such that the marginal value of waiting equals the marginal cost of delaying entry. The marginal cost is instantaneous profit foregone by waiting a differential unit of time: $\frac{A^{2} e^{\alpha t_{n}^{e}}}{(n+1)^{2}} d t$. In turn, the marginal value is sunk cost saved by delaying entry plus the marginal option value of waiting and avoiding an irreversible action.

as the lognormal distribution. In particular, letting $\alpha>\sup _{t} \frac{d \lambda(t) / d t}{\lambda(t)}$ would suffice. It is worthwhile pointing out that the results would continue to hold under such assumption.

19 The proof of the remark follows from straightforward manipulations that yield that this expression can be rewritten as $e^{\alpha t_{n}^{e}}\left(1+\frac{\alpha}{r+\lambda\left(t_{n}^{e}\right)}\right)=\frac{K(\alpha+r)(n+1)^{2}}{A^{2}}$. 
Thus, firm $n$ believes that the demand of the product may suddenly decay right after time $t_{n}^{e}$, so by waiting it believes that it eludes making a negative payoff with probability $\lambda\left(t_{n}^{e}\right) d t .^{20}$ Overall, the marginal value is equal to:

$$
r K d t+\lambda\left(t_{n}^{e}\right)\left(K-\int_{t_{n}^{e}}^{\infty} \frac{A^{2} e^{\alpha\left(2 t_{n}^{e}-s\right)}}{(n+1)^{2}} e^{-r\left(s-t_{n}^{e}\right)} d s\right) d t .
$$

In addition, the proof of Lemma 2 shows that $V_{n}(\cdot)$ is quasi-concave. More precisely, we note the following:

Remark 2: $t_{n}^{*}=0$ if and only if $V_{n}(\cdot)$ is monotonically decreasing on $[0, \infty)$, or, equivalently, $t_{n}^{*}=t_{n}^{e}$ if and only if $V_{n}(\cdot)$ is upward-sloping $\forall t_{n} \in\left(0, t_{n}^{e}\right)$ and downwardsloping $\forall t_{n} \in\left(t_{n}^{e}, \infty\right){ }^{21}$

After having examined firm $n$ 's optimal entry strategy when demand is still growing, we solve the remainder of the game for all of its predecessors, taking into account that the latter know the entry threshold of subsequent entrants. So let us analyze firm $n-1$ 's optimization problem now. We will assume that $t_{n}^{*}=t_{n}^{e}$ (because the solution to firm $n-1$ 's problem is $t_{n-1}^{*}=0$ whenever $t_{n}^{*}=0$, as shown by the proof of Lemma 4 below). $V_{n-1}(\cdot)$ can be shown to be continuously differentiable at $t_{n-1}^{\max }$, so it suffices to show that it is quasiconcave with its global maximum smaller than $t_{n-1}^{\max }$. Furthermore, we will only pay attention to the properties of $V_{n-1}(\cdot)$ on the region $\left[0, t_{n}^{e}\right)$, on which firm $n-1$ 's payoff function is:

\footnotetext{
${ }^{20}$ This follows from Lemma 1, which implies that the NPV of firm $n$ if it invested at date $t$ is negative if demand suddenly decays $\forall t<t_{n}^{\max }$.

${ }^{21}$ Not much can be said about the sign of the second derivative of the function (in fact, it can vary almost freely), although this is not relevant since it is not necessary throughout the analysis.
} 


$$
\begin{aligned}
V_{n-1}\left(t_{n-1}\right)= & \int_{t_{n-1}}^{t_{n}^{*}} f(\tau)\left(\int_{t_{n-1}}^{\infty} \pi(n-1, s, \tau) e^{-r s} d s-K e^{-r t_{n-1}}\right) d \tau \\
& \int_{t_{n}^{*}}^{\infty} f(\tau)\left(\int_{t_{n-1}^{*}}^{t_{n}^{*}} \pi(n-1, s, \tau) e^{-r s} d s+\int_{t_{n}^{*}}^{\infty} \pi(n, s, \tau) e^{-r s} d s-K e^{-r t_{n-1}}\right) d \tau
\end{aligned}
$$

If firm $n-1$ waits until $t_{n-1}$, then, for realizations larger than $t_{n-1}$ but smaller than $t_{n}^{*}$, it would gain oligopolistic profits indefinitely (for $n-1$ companies). If the cycle is believed to last enough so as to allow firm $n$ 's entry, then firm $n-1$ will expect to gain oligopolistic profits for $n-1$ firms until $t_{n}^{*}$, and oligopolistic profits for $n$ firms thereafter. Again we solve a relaxed program, replacing the constraint $t_{n-1} \in\left[t_{n-2}^{*}, t_{n}^{*}\right]$ with $t_{n-1} \geq 0$ :

$$
\begin{aligned}
& \max _{t_{n-1}} V_{n-1}\left(t_{n-1}\right) \\
& \text { s.t. } \quad t_{n-1} \geq 0
\end{aligned}
$$

Lemma 3 indicates firm $n-1$ 's optimal entry threshold, functionally very similar to that of firm $n$. The reason is that firm $n-1$ perceives that firm $n$ can take a fixed part of its rents with certain probability, so that entry by the latter is not marginally relevant. ${ }^{22}$

Lemma 3: Firm $n-1$ 's optimal strategy is "enter at $t=t_{n-1}^{e}$ if $t \leq \tau$; else, do not enter" if there exists a unique $t_{n-1}^{e} \in\left(0, t_{n-1}^{\max }\right)$ such that

$$
e^{\alpha t_{n-1}^{e}}\left(1+\frac{\alpha}{r+\lambda\left(t_{n-1}^{e}\right)}\right)=\frac{K(\alpha+r) n^{2}}{A^{2}} .
$$

Firm $n-1$ 's optimal strategy is "enter at $t=0$ if $t \leq \tau$; else, do not enter" if $t_{n-1}^{e}$ does not exist.

\footnotetext{
${ }^{22}$ Since $V_{n-1}\left(t_{n-1}\right)$ can be rewritten as
}

$$
\begin{aligned}
V_{n-1}\left(t_{n-1}\right)= & \int_{t_{n-1}}^{\infty} f(\tau)\left(\int_{t_{n-1}}^{\infty} \pi(n-1, s, \tau) e^{-r s} d s-K e^{-r t_{n-1}}\right) d \tau- \\
& \int_{t_{n}^{*}}^{\infty} f(\tau)\left(\int_{t_{n}^{*}}^{\infty}[\pi(n-1, s, \tau)-\pi(n, s, \tau)] e^{-r s} d s\right) d \tau
\end{aligned}
$$




\section{Proof: See Appendix.}

Finally, we use the same procedure to solve for firm $i$ 's optimal entry strategy, $i=1, \ldots, n-2$. For these cases, recursiveness allows us to prove Proposition 1, which summarizes the optimal entry dates for all firms: ${ }^{23}$

Proposition 1: Firm i's optimal strategy is "enter at $t=t_{i}^{e}$ if $t \leq \tau$; else, do not enter" if there exists a unique $t_{i}^{e} \in\left(0, t_{i}^{\max }\right)$ such that

$$
e^{\alpha t_{i}^{e}}\left(1+\frac{\alpha}{r+\lambda\left(t_{i}^{e}\right)}\right)=\frac{K(\alpha+r)(i+1)^{2}}{A^{2}} .
$$

Firm i's optimal strategy is "enter at $t=0$ if $t \leq \tau$; else, do not enter" if $t_{i}^{e}$ does not exist.

\section{Proof: See Appendix.}

However, it remains to check that our solution procedure is correct. Lemma 4 proves it.

Lemma 4: $t_{i}^{*}$ solves the corresponding constrained optimization program, so $t_{i}^{*} \geq t_{i-1}^{*} \forall i=2, \ldots, n$.

\section{Proof: See Appendix.}

We can now draw a series of straightforward results from Proposition 1. Noticing that, if $t_{i}^{e}$ exists, then $t_{i+1}^{e}$ exists $(i=1, \ldots, n-1)$, we have the following corollary:

Corollary 1: (a) If $t_{1}^{*}=t_{1}^{e}>0$, then $t_{j}^{*}=t_{j}^{e}>t_{i}^{*}=t_{i}^{e} \forall j>i \geq 1$. (b) If $V_{1}(0)<0$, then, $t_{i}^{*}=t_{i}^{e}>0 \forall i=1, \ldots, n$

The first part points out that, if firm 1, the pioneer, finds it more profitable to wait, then the followers must wait even longer until the market is sufficiently large to accommodate

\footnotetext{
${ }^{23}$ Although we do not write the functional form of $V_{i}\left(t_{i}\right)$ on the region $\left[0, t_{i+1}^{e}\right)$ in order to avoid a messy expression that is constructed in the same way as $V_{n-1}\left(t_{n-1}\right)$, it is straightforward to prove both Lemma 3 and Proposition 1 using the marginal interpretation that gives rise to Remark 1.
} 
them, since instantaneous profit are lower as more firms enter. In turn, the second part means that waiting may be valuable for all firms, no matter how many, even though the pioneer's net present value to immediate entry into the market were negative.

We also identify a necessary and sufficient condition for precommitment equilibria to be asymmetric, and provide a characterization of the firms' equilibrium payoffs (as in Reinganum (1981)):

Corollary 2: (a) Equilibria are asymmetric if and only if $t_{n}^{*}>0$ (i.e., $t_{n}^{e}$ exists). (b) The firms' equilibrium expected payoffs are non-increasing in the position of entry.

Proof: (a) Sufficiency is straightforward, whereas necessity follows from the second part of Corollary 1 and the fact that entry at $t_{n}^{*}=t_{n}^{e}>0$ is dominated for the first $n-1$ firms.

The first part of Corollary 2 is particularly relevant for empirical work because it shows that entry may be dispersed over time even when firms have no differing capabilities and they are totally symmetric, which contradicts common wisdom (see, e.g., Klepper and Graddy (1990, p. 37)).

On the other hand, Proposition 1 also allows for an analysis of how the value of firm $i$ 's investment opportunity depends on initial market size $A . t_{i}^{*}=0$ if and only if $t_{i}^{e}$ does not exist, so that $t_{i}^{*}=0$ if and only if $A \geq A_{i}^{*}=\left[\frac{K(\alpha+r)(i+1)^{2}}{1+\frac{\alpha}{r+f(0)}}\right]^{\frac{1}{2}}, \quad$ given that $\lim _{t \downarrow 0} \lambda(t)=\frac{\lim _{t \downarrow 0} f(t)}{\lim _{t \downarrow 0} \int_{t}^{\infty} f(\tau) d \tau}=\lim _{t \downarrow 0} f(t) \equiv f(0)$. Thus, $A_{i}^{*}$ is the initial market size that triggers immediate investment by firm $i$, obviously with $A_{1}^{*}<\ldots<A_{n}^{*}$. It summarizes all the factors taken into account by an immediate entrant: the growth/decay rate of the market $(\alpha)$, the number of incumbents including itself $(i)$, the degree of irreversibility of the decision $(K)$, 
the discount rate $(r)$, and the probability of instantaneous failure $(f(0))$. Note that an increase in the growth/decay rate of the market makes entry at date 0 more difficult for firm $i$, which means that firms care more about the risks of the market than the opportunities it offers. ${ }^{24}$

Based on the firms' equilibrium strategies, Propositions 2-4 fully characterize the patterns of entry over the PLC.

Proposition 2: If $A<A_{1}^{*}$, then $t_{i}^{*}=t_{i}^{e}>0 \forall i=1, \ldots, n$.

So for products whose current market is very small, firms would rather not enter immediately and would follow a "wait-and-see" approach. As a result, one may never see entry into that market, or, if demand grows sufficiently over time, entry may occur in a sequential fashion, with firms entering as the market gradually expands until its decay.

Proposition 3: If $A_{i}^{*} \leq A<A_{i+1}^{*}$ for some $i=1, \ldots, n-1$, then $t_{j}^{*}=0 \forall j=1, \ldots, i$ and $t_{j}^{*}=t_{j}^{e}>0 \forall j=i+1, \ldots, n$.

Hence, products with a sufficiently large demand may be simultaneously introduced by one or many firms at date 0 and then no more firms if the product is a failure. Otherwise, more firms may enter sequentially attracted by a successful product.

Proposition 4: If $A_{n}^{*} \leq A$, then $t_{i}^{*}=0 \forall i=1, \ldots, n$.

In turn, Proposition 4 implies that for products whose current market is extremely large, we would expect all firms entering at the same date 0 , no matter how successful the product turned out to be in the future.

${ }^{24} A_{i}^{*}$ can be rewritten as $A_{i}^{*}=\left[\frac{K(r+f(0))(i+1)^{2}}{1+\frac{f(0)}{\alpha+r}}\right]^{\frac{1}{2}}$, so it follows that an increase in $\alpha$ increases $A_{i}^{*}$. 
Finally, it can also be shown that Proposition 1 implies that $t_{2}^{*}-t_{1}^{*}>t_{3}^{*}-t_{2}^{*}>\ldots>t_{n}^{*}-t_{n-1}^{*}$. From this set of inequalities it follows that entry rates would accelerate over the life cycle if neither firm entered at date 0 :

Proposition 5: If $A<A_{1}^{*}$ and the random variable has an exponential distribution with parameter $\lambda$, then $t_{i+1}^{*}-t_{i}^{*}<t_{i}^{*}-t_{i-1}^{*} \forall i=2, \ldots, n-1$.

Proof: See Appendix.

A stylized fact regarding the initial stages of entry into an industry is that the number of firms grows in a "convex" fashion (see, e.g., Tirole (1988, p. 402)). ${ }^{25}$ Proposition 5 provides a theoretical rationale for this stylized fact. The proposition also implies that, as the number of firms grows large, the difference in the times of entry may be observed to converge to 0 . Proposition 6 below explains what drives this result.

\section{EXTENSIONS}

In this section, we extend the model presented in Section 2 and concisely develop a general model that encompasses many different types of competitive situations that may take place among symmetric entrants, including instantaneous price competition in cooperative or noncooperative settings. Most of our results carry over to this more general setup.

Following Londregan (1990), let us assume that it is instantaneous profit that follows a life cycle. So we replace Assumptions 1 and 3 with the following one:

Assumption A: If $i$ firms are operating in the market at date $t$, then each makes an instantaneous profit of:

$$
\Pi(i, t, \tau)= \begin{cases}\pi_{i} \exp (\alpha t) & \text { if } 0 \leq t \leq \tau \\ \pi_{i} \exp [\alpha(2 \tau-t)] & \text { if } t>\tau\end{cases}
$$

\footnotetext{
${ }^{25}$ This is for the range of times in which the S-shaped inter-firm diffusion path is convex. See, e.g., stages 1 and 2 of new product industries in Gort and Klepper (1982).
} 
In addition, let us assume that instantaneous profit has the following properties: it is monotone decreasing in $n$ and $\pi_{n}>0$. Hence, either costs are zero or they are sufficiently low so as to prevent firms from exiting.

It can be shown through similar steps to the ones we have followed in section 3 that Propositions 2-4 still hold in this more general setup, since the expression in Proposition 1 can be replaced with:

$$
e^{\alpha t_{i}^{e}}\left(1+\frac{\alpha}{r+\lambda\left(t_{i}^{e}\right)}\right)=\frac{K(\alpha+r)}{\pi_{i}} .
$$

Regarding Proposition 5, the general version is as follows:

Proposition 6: Let $\tau$ be an exponential random variable with parameter $\lambda$. Then $t_{i+1}^{e}-t_{i}^{e}<t_{i}^{e}-t_{i-1}^{e} \forall i=2, \ldots, n-1$ if and only if $\frac{\pi_{i}-\pi_{i+1}}{\pi_{i}}<\frac{\pi_{i-1}-\pi_{i}}{\pi_{i-1}}$, while $t_{i+1}^{e}-t_{i}^{e}>t_{i}^{e}-t_{i-1}^{e}$ if and only if $\frac{\pi_{i}-\pi_{i+1}}{\pi_{i}}>\frac{\pi_{i-1}-\pi_{i}}{\pi_{i-1}}$.

The assumptions of exponential growth and decay, as well as the assumption of an exponentially distributed random variable for the length of the ascending phase of the PLC are important because they lead to a simple result by dropping time-dependence. The proposition implies that accelerating (decelerating) entry rates in our setting are due to the fact that the percentage reduction in individual profit due to the immediate follower's entry decreases (increases) with $i$. Decreasingness holds in models such as linear Cournot or Salop with linear or quadratic costs, as well as collusive settings in which firms evenly divide instantaneous monopoly profits. Therefore, our model suggests that one of the driving forces of S-shaped diffusion paths would be the existence of a unique $i^{*}$ such that $\frac{\pi_{i}-\pi_{i+1}}{\pi_{i}}<\frac{\pi_{i-1}-\pi_{i}}{\pi_{i-1}} \forall i \leq i^{*}$ and $\frac{\pi_{i}-\pi_{i+1}}{\pi_{i}}>\frac{\pi_{i-1}-\pi_{i}}{\pi_{i-1}} \forall i>i^{*}$. This would definitely require 
some type of critical change in the nature of product market competition due to entry by firm $i^{*}$.

\section{CONCLUSION}

The main purpose of this paper is to provide a theoretical framework that explains different dynamics of entry over the PLC. The driving factors of entry decisions are strategic interaction, uncertainty about future demand evolution and investment irreversibility. In this sense, we have examined conditions under which entry into a market may be simultaneous and/or sequential, with or without accelerating rates of entry. The results suggest that entry rates accelerate or decelerate depending on whether the percentage reduction in individual profit due to the immediate follower's entry decreases or increases with the number of remaining entrants. Whether or not demand factors, irreversibility and uncertainty about the PLC are relevant in explaining entry patterns is an empirical matter. Still, it seems interesting to test them versus inter-firm diffusion of technological knowledge and the rate of innovation within the industry.

The paper has some important limitations too, which would be worthwhile examining. We have assumed that the pattern of non-cumulative adoptions of the product by consumers is exogenous to the firms, but unknown. Specifically, the time of the peak is uncertain, as well as the adoption rate at the peak. The model can easily endogenize the distribution function governing the maturity date. This may be relevant if the activities of firms before entering the market can affect the properties of the PLC. For instance, let us consider a setting with symmetric firms and a random variable that is exponentially distributed with a hazard rate positively correlated to pre-entry activities of each of the firms. Under these assumptions, the open-loop equilibria we have described would have to consider the incentives of late entrants to free-ride on the efforts of earlier entrants. In principle, early entrants would benefit 
more from investing because their expected payoff is higher (by Corollary 2). However, their incentives to invest in increasing the hazard rate would lessen because their investment would also benefit later entrants, which would speed up entry by the latter. ${ }^{26}$ As a result, pioneers may underinvest relative to later entrants, so that it may happen that it is early entrants that free ride on late entrants' efforts.

Lastly, we have not studied exit decisions, which would take place if market size were small enough so as not to cover operating or opportunity costs, and may require imposing an exit order even without the symmetry assumption. The fact that firms would exit the market would delay entry in an unpredictable way, and would constitute a significant improvement of our understanding about the entry and exit dynamics over the PLC, and aspect that has been unexplored thus far.

\footnotetext{
${ }^{26}$ Effects of investment on own entry timing would be of second-order, while effects on later entrants' entry timing would be of first-order.
} 


\section{REFERENCES}

Barbarino, A. T. and Boyan Jovanovic (2003), "Shakeouts and Market Crashes", manuscript, Dept. of Economics, University of Chicago.

Bass, F.M. (1969), “A New Product Growth for Model Consumer Durables”, Management Science, 15(5): 215-227.

Bass, F.M. (1980), “The Relationship between Diffusion Rates, Experience Curves, and Demand Elasticities for Consumer Durable Technological Innovations”, Journal of Business, 53(3): S51-S67.

Bollen, N.P.B. (1999), "Real Options and Product Life Cycles", Management Science, 45(5): 670-684.

Brockhoff, K. (1967), “A Test for the Product Life Cycle”, Econometrica, 35(3-4): 472-484.

Dixit, A. K. and Robert S. Pindyck (1994), "Investment under Uncertainty", Princeton, NJ, Princeton University Press.

Dodson J. A. and Eitan Muller (1978), "Models of New Product Diffusion Through Advertising and Word-of-Mouth", Management Science, 24 (15):1568-1578.

Fudenberg, D. and Jean Tirole (1985), "Preemption and Rent Equalization in the Adoption of New Technology”, Review of Economic Studies, 52(3): 383-401.

Gort, M. and Steven Klepper (1982), "Time Paths in the Diffusion of Product Innovations", Economic Journal, 92(367): 630-653.

Gutiérrez Arnaiz O. and Francisco Ruiz-Aliseda (2003), "Real Options with Unknown-Date Events", The Center for Mathematical Studies in Economics and Management Science", Northwestern University, DP \# 1378.

Grant, R. M. (1998), “Contemporary Strategy Analysis: Concepts, Techniques and Applications", $3^{\text {rd }}$ Edition, Cambridge, MA, Basil Blackwell. 
Hopenhayn, H. A. (1992), "Entry, Exit and Firm Dynamics in Long Run Equilibrium”, Econometrica, 60(5): 1127-1150.

Jovanovic, B. (1982), "Selection and the Evolution of Industry", Econometrica, 50(3): 649670.

Klepper, S. and Elizabeth Graddy (1990), "The Evolution of New Industries and the Determinants of Market Structure”, Rand Journal of Economics 21(1): 27-44.

Kotler, P. (1999), "Marketing Management: Analysis, Planning and Control”, $10^{\text {th }}$ Edition, Englewood Cliffs, NJ, Prentice-Hall.

Kwoka, J. E. (1996), “Altering the Product Life Cycle of Consumer Durables: The Case of Minivans", Managerial and Decision Economics, 17(1): 17-25.

Lilien, G. L. and Eunsang Yoon (1990), "The Timing of Competitive Market Entry: An Exploratory Study of New Industrial Products”, Management Science, 36(5): 568-585.

Londregan, J. (1990), "Entry and Exit over the Industry Life Cycle", Rand Journal of Economics 21(3): 446-458.

Pisano, G. P. and Steven C. Wheelwright (1995), “The New Logic of High-tech R\&D”, Harvard Business Review, 73(5): 93-105.

Polli, R. and Victor Cook (1969), "Validity of the Product Life Cycle", Journal of Business, 42(4): 385-400.

Reinganum, J.F. (1981), "Market Structure and the Diffusion of New Technology", Bell Journal of Economics, 12(2): 618-624.

Tirole, J. (1988), “The Theory of Industrial Organization”, $1^{\text {st }}$ Edition, Cambridge, MA, MIT Press.

Tsurumi, H. and Yoshi Tsurumi (1980), "A Bayesian Test of the Product Life Cycle Hypothesis as Applied to the U.S. Demand for Color-TV Sets", International Economic Review, 21(3): 583-597. 


\section{LIST OF FIGURES}

Figure 1: Two possible realizations of the proposed PLC

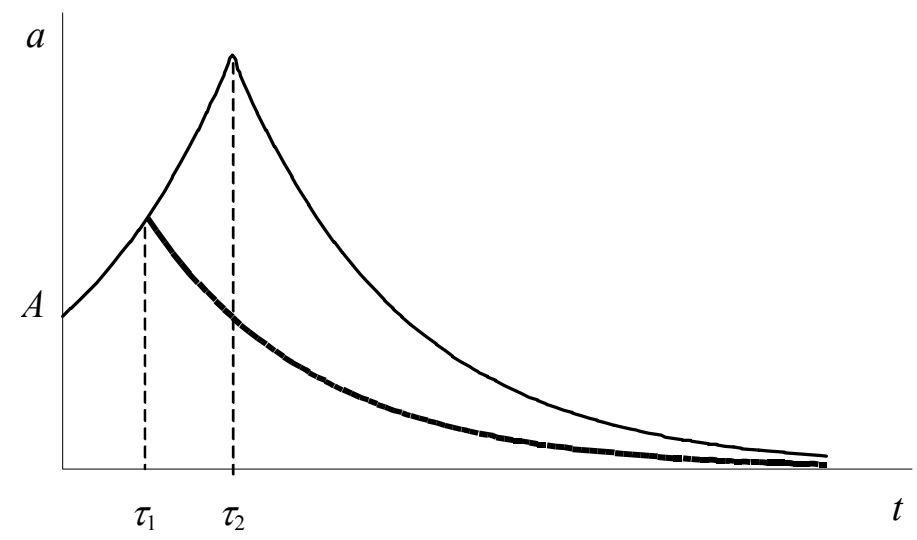




\section{APPENDIX}

Proof of Lemma 1: Suppose that $0 \leq j<n$ firms had entered the market during the growth phase and suddenly $\tau$ were revealed. By relabeling the remaining firms (as well as the associated flow of profits), we can let $j=0$ without loss of generality. Denoting firms' entry times by $t_{i}^{d}$, let us solve for firm $n$ 's entry problem, knowing that it cannot enter before $t_{n-1}^{d} \geq \tau$.

First we prove that, if immediate investment at $t_{n-1}^{d}$ is not preferred to delayed investment at some $\hat{t}_{n}>t_{n-1}^{d}$, then $N P V_{n}\left(\hat{t}_{n}\right)=\int_{\hat{t}_{n}}^{\infty}\left(\frac{A}{n+1}\right)^{2} e^{2 \alpha \tau} e^{-(\alpha+r) s} d s-K e^{-r \hat{t}_{n}} \leq 0$.

Thus, note that $N P V_{n}\left(t_{n}\right)=\int_{t_{n}}^{\infty}\left(\frac{A}{n+1}\right)^{2} e^{2 \alpha \tau} e^{-(\alpha+r) s} d s-K e^{-r t_{n}}$ is a strictly quasi-convex function on $\quad(-\infty,+\infty),{ }^{27} \quad$ with $\quad \lim _{t_{n} \rightarrow \infty} N P V_{n}\left(t_{n}\right)=0 \quad$ and $\bar{t}_{n} \equiv \underset{t_{n}}{\arg \min } N P V_{n}\left(t_{n}\right)=2 \tau+\frac{1}{\alpha} \log \left(\frac{A^{2}}{r K(n+1)^{2}}\right)$. It suffices to show that the contrapositive of the claim is true, so assume that $N P V_{n}\left(\hat{t}_{n}\right)>0$. Because of strict quasi-convexity and existence of the global minimum, we must have that either $\underset{t_{n} \in\left[t_{n-1}^{d}, \infty\right]}{\arg \max } N P V_{n}\left(t_{n}\right)=\infty$ or $\underset{t_{n} \in\left[\left[_{n-1}^{d}, \infty\right]\right.}{\arg \max } N P V_{n}\left(t_{n}\right)=t_{n-1}^{d}$. In the first case, the fact that $\lim _{t_{n} \rightarrow \infty} N P V_{n}\left(t_{n}\right)=0$ would contradict the assumption that $N P V_{n}\left(\hat{t}_{n}\right)>0$, by the definition of maximum. Hence, we must have that $\underset{t_{n} \in\left[t_{n-1}^{d}, \infty\right]}{\arg \max } N P V_{n}\left(t_{n}\right)=t_{n-1}^{d}$. But then $N P V_{n}\left(t_{n-1}^{d}\right)>N P V_{n}\left(\hat{t}_{n}\right)$. This shows that the contrapositive is true, and thus we must have $t_{n}^{d}=t_{n-1}^{d}$. Repeating this procedure, it is easy to show that $t_{i}^{d}=\tau \forall i$. Given such entry behavior by all firms, it is clear that our assumption

${ }^{27}$ Formally, because $\frac{d N P V_{n}\left(t_{n}\right)}{d t_{n}}=0 \Rightarrow \frac{d^{2} N P V_{n}\left(t_{n}\right)}{d t_{n}^{2}}>0$. 
that the entry order of firms is based on their index acts as a coordination device: if there is room only for $i$ firms, then only the first $i$ firms enter (not the last ones, for instance).

So it remains to show that immediate investment at $\tau$ by firm $i$ is profitable if and only if $\tau \geq t_{i}^{\max }$, which will complete the entire proof. It clearly suffices to show that firm $i$ 's payoff if it invested at $t_{i}^{\max }$ is zero, since $N P V_{i}(\tau)>0 \forall \tau>t_{i}^{\max } \cdot{ }^{28}$ Let $t_{i}^{\max }>0$ and note that

$$
N P V_{i}\left(t_{i}^{\max }\right)=\int_{t_{i}^{\max }}^{\infty}\left(\frac{A}{n+1}\right)^{2} e^{2 \alpha t_{i}^{\max }} e^{-(\alpha+r) s} d s=\frac{A^{2} e^{(\alpha-r) t_{i}^{\max }}}{(\alpha+r)(n+1)^{2}}-K e^{-r t_{i}^{\max }}=0 .
$$

If $t_{i}^{\max }=0$, then $N P V_{i}(0)=\frac{A^{2}-(\alpha+r) K(n+1)^{2}}{(\alpha+r)(n+1)^{2}}=0$. This completes the proof.

Proof of Lemma 2: We will first show that $V_{n}\left(t_{n}\right)$ is monotone decreasing if $t_{n}^{\max }=0$. In this case, the function becomes:

$$
\begin{aligned}
V_{n}\left(t_{n}\right)= & \int_{t_{n}^{\max }}^{t_{n}} f(\tau)\left(\int_{\tau}^{\infty} \pi(n, s, \tau) e^{-r s} d s-K e^{-r \tau}\right) d \tau+\int_{t_{n}}^{\infty} f(\tau)\left(\int_{t_{n}}^{\infty} \pi(n, s, \tau) e^{-r s} d s-K e^{-r t_{n}}\right) d \tau= \\
& \int_{t_{n} \operatorname{tax}}^{t_{n}} f(\tau)\left(\int_{\tau}^{\infty}\left(\frac{A}{n+1}\right)^{2} e^{2 \alpha \tau} e^{-(\alpha+r) s} d s-K e^{-r \tau}\right) d \tau+ \\
& \int_{t_{n}}^{\infty} f(\tau)\left(\int_{t_{n}}^{\tau}\left(\frac{A}{n+1}\right)^{2} e^{(\alpha-r) s} d s+\int_{\tau}^{\infty}\left(\frac{A}{n+1}\right)^{2} e^{2 \alpha \tau} e^{-(\alpha+r) s} d s-K e^{-r t_{n}}\right) d \tau .
\end{aligned}
$$

Differentiating it with respect to $t_{n}$ and performing some algebraic manipulations yields:

$$
\frac{d V_{n}\left(t_{n}\right)}{d t_{n}} \equiv V_{n}^{\prime}\left(t_{n}\right)=\left[r K-\left(\frac{A}{n+1}\right)^{2} e^{\alpha t_{n}}\right] e^{-r t_{n}} \int_{t_{n}}^{\infty} f(\tau) d \tau
$$

We claim that $V_{n}^{\prime}\left(t_{n}\right)<0$ for $t_{n} \geq 0$. Otherwise, we would reach a contradiction:

${ }^{28}$ The proof is as follows. Let $\delta>0$. Then:

$$
\begin{aligned}
N P V_{i}\left(t_{i}^{\max }+\delta\right)= & \frac{A^{2} e^{(\alpha-r)\left(t_{i}^{\max }+\delta\right)}}{(\alpha+r)(n+1)^{2}}-K e^{-r\left(t_{i}^{\max }+\delta\right)}=e^{-r \delta}\left(\frac{A^{2} e^{(\alpha-r) t_{i}^{\max }} e^{\alpha \delta}}{(\alpha+r)(n+1)^{2}}-K e^{-r t_{i}^{\max }}\right)= \\
& e^{-r \delta\left(e^{\alpha \delta} K e^{-r t_{i}^{\max }}-K e^{-r t_{i}^{\max }}\right)>0}
\end{aligned}
$$

where the last equality follows from the definition of $t_{i}^{\max }$. 


$$
0 \leq\left[r K-\left(\frac{A}{n+1}\right)^{2} e^{\alpha t_{n}}\right] e^{-r t_{n}} \int_{t_{n}}^{\infty} f(\tau) d \tau \leq-\alpha K e^{-r t_{n}} \int_{t_{n}}^{\infty} f(\tau) d \tau<0
$$

since $t_{n}^{\max }=0$ if and only if $r K+\alpha K \leq\left(\frac{A}{n+1}\right)^{2}$. Given that Assumption 5 implies that $V_{n}\left(t_{n}\right)$ is bounded above, this shows that $V_{n}\left(t_{n}\right)$ attains a unique global maximum when $t_{n}^{\max }=0$

To conclude the proof, let $t_{n}^{\max }>0$. Now note that $V_{n}\left(t_{n}\right)$ can be rewritten as follows:

$$
\begin{aligned}
V_{n}\left(t_{n}\right)= & \int_{t_{n}}^{\infty} f(\tau)\left(\int_{t_{n}}^{\infty} \pi(n, s, \tau) e^{-r s} d s-K e^{-r t_{n}}\right) d \tau= \\
& \int_{t_{n}}^{\infty} f(\tau)\left[\int_{t_{n}}^{\tau}\left(\frac{A}{n+1}\right)^{2} e^{(\alpha-r) s} d s+\int_{\tau}^{\infty}\left(\frac{A}{n+1}\right)^{2} e^{2 \alpha \tau} e^{-(\alpha+r) s} d s-K e^{-r t_{n}}\right] d \tau
\end{aligned}
$$

By Assumption 5, this function is bounded above. Differentiating it with respect to $t_{n}$, solving the integrals, taking into account that $\lambda\left(t_{n}\right)=\frac{f\left(t_{n}\right)}{\int_{t_{n}}^{\infty} f(\tau) d \tau}$ and rearranging yields:

$$
\begin{aligned}
\frac{d V_{n}\left(t_{n}\right)}{d t_{n}} \equiv & V_{n}^{\prime}\left(t_{n}\right)=-\frac{A^{2}}{(\alpha+r)(n+1)^{2}} e^{(\alpha-r) t_{n}} f\left(t_{n}\right)-\frac{A^{2}}{(n+1)^{2}} e^{(\alpha-r) t_{n}} \int_{t_{n}}^{\infty} f(\tau) d \tau+ \\
& K^{-r t_{n}}\left(f\left(t_{n}\right)+r \int_{t_{n}}^{\infty} f(\tau) d \tau\right)= \\
& \left(-\frac{A^{2}}{(\alpha+r)(n+1)^{2}} e^{\alpha t_{n}} \lambda\left(t_{n}\right)-\frac{A^{2}}{(n+1)^{2}} e^{\alpha t_{n}}+K\left(r+\lambda\left(t_{n}\right)\right)\right) \int_{t_{n}}^{\infty} e^{-r t_{n}} f(\tau) d \tau
\end{aligned}
$$


If an interior maximum $t_{n}^{e}$ exists, it must be such that $V_{n}^{\prime}\left(t^{e}\right)=0$. Then multiplying through by $\frac{(\alpha+r)(n+1)^{2}}{\infty}$ and rearranging, the first-order condition can be written as follows: ${ }^{29}$ $A^{2} \int_{t^{e}}^{\infty} e^{-r t^{e}} f(\tau) d \tau$

$$
e^{\alpha t_{n}^{e}}\left(1+\frac{\alpha}{r+\lambda\left(t_{n}^{e}\right)}\right)=\frac{K(\alpha+r)(n+1)^{2}}{A^{2}}
$$

So if such $t_{n}^{e}$ exists, then it must be unique because the hazard-rate is non-increasing (the result follows from the intermediate value theorem and expression (A1)). Also, if $t_{n}^{e}$ exists, then $t_{n}^{e}<t_{n}^{\max }$. Otherwise, given that $t_{n}^{e}>0$, a contradiction would obtain using (A1) and the fact that $\frac{\alpha}{r+\lambda\left(t_{n}^{e}\right)}>0$ :

$$
\begin{aligned}
& t_{n}^{e} \geq t_{n}^{\max }=\frac{1}{\alpha} \log \left(\frac{K(\alpha+r)(n+1)^{2}}{A^{2}}\right)=\frac{1}{\alpha} \log \left[e^{\alpha t_{n}^{e}}\left(1+\frac{\alpha}{r+\lambda\left(t_{n}^{e}\right)}\right)\right] \Rightarrow \\
& e^{\alpha t_{n}^{e}} \geq e^{\alpha t_{n}^{e}}\left(1+\frac{\alpha}{r+\lambda\left(t_{n}^{e}\right)}\right) .
\end{aligned}
$$

Now we check that $V_{n}^{\prime \prime}\left(t_{n}^{e}\right)<0$, so that $t_{n}^{e}$ is indeed a global maximizer whenever it exists.

Given that

$$
V_{n}^{\prime}\left(t_{n}\right)=\left(-\frac{A^{2}}{(\alpha+r)(n+1)^{2}} e^{\alpha t_{n}} \lambda\left(t_{n}\right)-\frac{A^{2}}{(n+1)^{2}} e^{\alpha t_{n}}+K\left(r+\lambda\left(t_{n}\right)\right)\right) \int_{t_{n}}^{\infty} e^{-r t_{n}} f(\tau) d \tau,
$$

we have:

\footnotetext{
${ }^{29}$ If $\alpha=r$, then we should derive the functional form of $V_{n}\left(t_{n}\right)$ from scratch, since directly plugging in $\alpha=r$ implies that $V_{n}\left(t_{n}\right)$ is not well defined. Yet, straightforward calculations show that the same expression obtains for computing $t_{n}^{e}$.
} 


$$
\begin{aligned}
V_{n}^{\prime \prime}\left(t_{n}^{e}\right)= & \left(-\frac{\alpha A^{2} e^{\alpha t_{n}^{e}} \lambda\left(t_{n}^{e}\right)}{(\alpha+r)(n+1)^{2}}-\frac{A^{2} e^{\alpha t_{n}^{e}} \lambda^{\prime}\left(t_{n}^{e}\right)}{(\alpha+r)(n+1)^{2}}-\frac{\alpha A^{2} e^{\alpha t_{n}^{e}}}{(n+1)^{2}}+K \lambda^{\prime}\left(t_{n}^{e}\right)\right) \int_{t_{n}^{e}}^{\infty} e^{-r t_{n}^{e}} f(\tau) d \tau- \\
& \left(f\left(t_{n}^{e}\right) e^{-r t_{n}^{e}}+\int_{t_{n}^{e}}^{\infty} r e^{-r t_{n}^{e}} f(\tau) d \tau\right)\left(-\frac{A^{2} e^{\alpha t_{n}^{e}} \lambda\left(t_{n}^{e}\right)}{(\alpha+r)(n+1)^{2}}-\frac{A^{2} e^{\alpha t_{n}^{e}}}{(n+1)^{2}}+K\left(r+\lambda\left(t_{n}^{e}\right)\right)\right)= \\
& {\left[-\frac{\alpha A^{2} e^{\alpha t_{n}^{e}} \lambda\left(t_{n}^{e}\right)}{(\alpha+r)(n+1)^{2}}-\frac{\alpha A^{2} e^{\alpha t_{n}^{e}}}{(n+1)^{2}}+\lambda^{\prime}\left(t_{n}^{e}\right)\left(K-\frac{A^{2} e^{\alpha t_{n}^{e}}}{(\alpha+r)(n+1)^{2}}\right)\right]_{t_{n}^{e}}^{\infty} e^{-r t_{n}^{e}} f(\tau) d \tau, }
\end{aligned}
$$

where we have made use of (A1). Given that $\lambda^{\prime}\left(t_{n}^{e}\right) \leq 0$ (we assume differentiability for convenience, although it is not necessary) and $K>\frac{A^{2} e^{\alpha t_{n}^{e}}}{(\alpha+r)(n+1)^{2}}$ because $t_{n}^{e}<t_{n}^{e}$, it follows that $V_{n}^{\prime \prime}\left(t_{n}^{e}\right)<0$.

$t_{n}^{e}$ may not exist, though. Then we claim that $V_{n}(\cdot)$ must be monotone decreasing. To prove it, first note that $V_{n}\left(t_{n}^{\max }\right)>0$ by definition of $t_{n}^{\max }$. Second, it can be shown that $V_{n}(\cdot)$ is monotone decreasing on the set $\left[t_{n}^{\max }, \infty\right)$, since, on this region, we have:

$$
V_{n}\left(t_{n}\right)=\int_{t_{n}^{\max }}^{t_{n}} f(\tau)\left(\int_{\tau}^{\infty} \pi(n, s, \tau) e^{-r s} d s-K e^{-r \tau}\right) d \tau+\int_{t_{n}}^{\infty} f(\tau)\left(\int_{t_{n}}^{\infty} \pi(n, s, \tau) e^{-r s} d s-K e^{-r t_{n}}\right) d \tau
$$

Recall that we showed at the beginning of the proof that

$$
V_{n}^{\prime}\left(t_{n}\right)=\left(r K-\frac{A^{2} e^{\alpha t_{n}}}{(n+1)^{2}}\right) e^{-r t_{n}} \int_{t_{n}}^{\infty} f(\tau) d \tau
$$

Letting $t_{n}=t_{n}^{\max }+\delta$, where $\delta \geq 0$, we must have that $V_{n}^{\prime}\left(t_{n}^{\max }+\delta\right)<0$. Otherwise, the fact that $e^{\alpha \delta} \geq 1$ would lead us to the following contradiction:

$$
\begin{aligned}
& 0 \leq\left(r K-\frac{A^{2} e^{\alpha t_{n}^{\max }} e^{\alpha \delta}}{(n+1)^{2}}\right) e^{-r\left(t_{n}^{\max }+\delta\right)} \int_{t_{n}^{\max }+\delta}^{\infty} f(\tau) d \tau= \\
& \left(r K-r K e^{\alpha \delta}-\alpha K e^{\alpha \delta}\right) e^{-r\left(t_{n}^{\max }+\delta\right)} \int_{t_{n}^{\max }+\delta}^{\infty} f(\tau) d \tau<0 .
\end{aligned}
$$

Now it suffices to prove that $V_{n}(\cdot)$ is monotone decreasing on $\left[0, t_{n}^{\max }\right)$ if $t_{n}^{e}$ does not exist. We will do it by contradiction. Consider first the case in which $V_{n}(0) \geq V_{n}\left(t_{n}^{\max }\right)$. On the one 
hand, note that if $V_{n}(\cdot)$ is not monotonically decreasing on $\left[0, t_{n}^{\max }\right)$, then we cannot have $\frac{d V_{n}}{d t_{n}}(0)>0$. Otherwise, the fact that $V_{n}(0) \geq V_{n}\left(t_{n}^{\max }\right)$ implies that there must exist one critical point $\bar{t}_{n} \in\left(0, t_{n}^{\max }\right)$ at which $\frac{d V_{n}}{d t_{n}}\left(\bar{t}_{n}\right)<0$, given the (continuity and) differentiability of $V_{n}(\cdot)$. Because $\frac{d V_{n}}{d t_{n}}(0)>0$, it follows from the continuous differentiability of $V_{n}(\cdot)$ that $\frac{d V_{n}}{d t_{n}}\left(\hat{t}_{n}\right)=0$ for some $\hat{t}_{n} \in\left(0, \bar{t}_{n}\right)$. The definition and uniqueness of $t_{n}^{e}$ imply that $\hat{t}_{n}=t_{n}^{e}$, which contradicts the non-existence of $t_{n}^{e}$. On the other hand, if $V_{n}(\cdot)$ were not monotonically decreasing and $\frac{d V_{n}}{d t_{n}}(0) \leq 0$, then exactly the same reasoning as before would lead to the existence of a critical point (a minimum in this case), which cannot be either. (The case in which $V_{n}(0)=V_{n}\left(t_{n}^{\max }\right)$ follows from the fact that the derivative of $V_{n}(\cdot)$ is clearly not equal to $0 \quad \forall t_{n} \in\left[0, t_{n}^{\max }\right)$.) Hence, $V_{n}(\cdot)$ must be monotonically decreasing if $V_{n}(0) \geq V_{n}\left(t_{n}^{\max }\right)$. Therefore, a unique global maximum exists because $V_{n}(\cdot)$ is bounded. Now suppose that $V_{n}(\cdot)$ is not monotonically decreasing on $\left[0, t_{n}^{\max }\right)$, with $V_{n}(0)<V_{n}\left(t_{n}^{\max }\right)$. Then the continuity and differentiability of $V_{n}(\cdot)$ imply that there must be some $\bar{t}_{n} \in\left(0, t_{n}^{\max }\right)$ such that $\frac{d V_{n}}{d t_{n}}\left(\bar{t}_{n}\right)>0$. Given that $\frac{d V_{n}}{d t_{n}}\left(t_{n}^{\max }\right)<0$, it follows that $\frac{d V_{n}}{d t_{n}}\left(\hat{t}_{n}\right)=0$ for some $\hat{t}_{n} \in\left(\bar{t}_{n}, t_{n}^{\max }\right.$ ) (by the continuous differentiability of $\left.V_{n}(\cdot)\right)$. So we must have that such $\hat{t}_{n}$ is $t_{n}^{e}$, a contradiction. All this shows that we indeed have that $V_{n}(\cdot)$ must be monotone decreasing whenever $t_{n}^{e}$ does not exist, and thus $V_{n}(\cdot)$ attains a unique global maximum at date 0 . 
Proof of Lemma 3: The only difference with Lemma 2 is the determination of $t_{n-1}^{e}$, so we next show how it is derived. First note that $V_{n-1}\left(t_{n-1}\right)$ can rewritten as a function of $V_{n}\left(t_{n}^{*}\right)$ after some simple manipulations:

$$
\begin{aligned}
V_{n-1}\left(t_{n-1}\right)= & \int_{t_{n-1}}^{\infty} f(\tau)\left(\int_{t_{n-1}}^{\infty} \pi(n-1, s, \tau) e^{-r s} d s-K e^{-r t_{n-1}}\right) d \tau- \\
& \int_{t_{n}^{*}}^{\infty} f(\tau)\left(\int_{t_{n}^{*}}^{\infty}(\pi(n-1, s, \tau)-\pi(n, s, \tau)) e^{-r s} d s\right) d \tau= \\
& \int_{t_{n-1}}^{\infty} f(\tau)\left(\int_{t_{n-1}}^{\infty} \pi(n-1, s, \tau) e^{-r s} d s-K e^{-r t_{n-1}}\right) d \tau+V_{n}\left(t_{n}^{*}\right)- \\
& \int_{t_{n}^{*}}^{\infty} f(\tau)\left(\int_{t_{n}^{*}}^{\infty} \pi(n-1, s, \tau) e^{-r s} d s-K e^{-r t_{n}^{*}}\right) d \tau
\end{aligned}
$$

The value of firm $n-1$ 's investment opportunity nests the value of the immediate follower (a constant from its viewpoint) so that its maximand is structurally similar to that of the follower plus a constant. Hence, its solution must be the same, mutatis mutandis:

$$
e^{\alpha t_{n-1}^{e}}\left(1+\frac{\alpha}{r+\lambda\left(t_{n-1}^{e}\right)}\right)=\frac{K(\alpha+r) n^{2}}{A^{2}}
$$

Proof of Proposition 1: The proposition is true for firms $n-1$ and $n$ by Lemmas 2 and 3, and we only briefly sketch the formal argument, very similar to proof of Lemma 3 . The point is that firm $i$ 's problem is identical to that solved by its followers, so its solution must be identical, mutatis mutandis.

Proof of Lemma 4: We first show that $t_{n}^{*}=0$ implies $t_{1}^{*}=0$. It is easy to see that $t_{n}^{*}=0$ implies $t_{n-1}^{*}=0$, since, otherwise, $t_{n-1}^{e}$ would exist (by Lemma 3), from where it would trivially follow that $t_{n}^{e}$ also exists, as $\frac{K(\alpha+r) n^{2}}{A^{2}}<\frac{K(\alpha+r)(n+1)^{2}}{A^{2}}$, contradicting the assumption that $t_{n}^{*}=0$. Similarly, $t_{n-1}^{*}=0$ implies $t_{n-2}^{*}=0$, and so on and so forth, which shows that $t_{1}^{*}=0$. Hence, $t_{i}^{*} \geq t_{i-1}^{*} \forall i=2, \ldots, n$, whenever $t_{n}^{*}=0$. 
The proof is a little bit more involved when $t_{n}^{*}=t_{n}^{e}$, so we will refer interested readers to the proof of Proposition 2 in Reinganum (1981, p. 621), since it contains all the basic ingredients that are required.

Proof of Proposition 5: Suppose not. Then, $t_{i+1}^{e}-t_{i}^{e} \geq t_{i}^{e}-t_{i-1}^{e}$ for some $i=2, \ldots, n-1$. Note that the hazard rate of the exponential distribution is the constant $\lambda$ so straightforward calculations yield

$$
t_{i}^{e}=\frac{1}{\alpha} \log \left(\frac{K(\alpha+r)(r+\lambda)(i+1)^{2}}{A^{2}(\lambda+r+\alpha)}\right)
$$

If there exists an $i=2, \ldots, n-1$ such that $t_{i+1}^{e}-t_{i}^{e} \geq t_{i}^{e}-t_{i-1}^{e}$, then standard properties of natural logarithms yield that $2 t_{i}^{e} \leq t_{i-1}^{e}+t_{i+1}^{e}$ for such firm if and only if:

$$
\frac{1}{\alpha} \log \left[\left(\frac{K(\alpha+r)(r+\lambda)(i+1)^{2}}{A^{2}(\lambda+r+\alpha)}\right)^{2}\right] \leq \frac{1}{\alpha} \log \left[\left(\frac{K(\alpha+r)(r+\lambda)(i+2) i}{A^{2}(\lambda+r+\alpha)}\right)^{2}\right] .
$$

This is true if and only if $i(i+2) \geq(i+1)^{2}$, which cannot be

Proof of Proposition 6: $t_{i+1}^{e}-t_{i}^{e}<t_{i}^{e}-t_{i-1}^{e}$ if and only $t_{i-1}^{e}+t_{i+1}^{e}<2 t_{i}^{e}$ if and only if

$$
\frac{1}{\alpha} \log \left[\left(\frac{1}{\pi_{i}}\right)^{2}\left(\frac{K(\alpha+r)(r+\lambda)}{(\lambda+r+\alpha)}\right)^{2}\right]>\frac{1}{\alpha} \log \left[\left(\frac{1}{\pi_{i-1} \pi_{i+1}}\right)\left(\frac{K(\alpha+r)(r+\lambda)}{(\lambda+r+\alpha)}\right)^{2}\right]
$$

if and only if $\frac{\pi_{i+1}}{\pi_{i}}>\frac{\pi_{i}}{\pi_{i-1}}$ if and only if $\frac{\pi_{i}-\pi_{i-1}}{\pi_{i-1}}<\frac{\pi_{i+1}-\pi_{i}}{\pi_{i}}$. The remainder of the proof is trivial. 